\title{
NONLINEAR TEMPORAL MODEL FOR FORMATION OF PULSAR MICROSTRUCTURES
}

\author{
Abraham C.-L. Chian \\ Institute for Space Research-INPE
}

\begin{abstract}
A nonlinear plasma model which may account for temporal modulation of pulsar radio pulses is presented. Envelope solitons and envelope nonlinear wave trains can result from the nonlinear interaction of the high-frequency coherent pulsar radiation with the pulsar magnetosphere. Theories of electromagnetic envelope solitons and electromagnetic envelope nonlinear wave trains in electronpositron plasmas are reviewed. The application of this model for observation of pulsar microstructures is discussed.
\end{abstract}

\section{Introduction}

Pulsar microstructures (or micropulses) are very rapid intensity variations in individual pulses from radio pulsars with time scales ranging from milliseconds to microseconds (Hankins and Boriakoff 1978, Cordes 1979, Cordes and Hankins 1979, Bartel, Sieber, and Graham 1980, Ferguson 1981, Bartel and Hankins 1982). These microstructures are the finest features of single pulsar pulses, hence they should hold important clues about the physical processes of generation and propagation of pulsar radio emission.

A nonlinear temporal model for formation of pulsar microstrurtures was first proposed by Chian and Kennel (1983), wherin it was shown that the intensity of pulsar radio emission in the source region can be measured by the wave strength parameter

$$
\nu_{\mathrm{E}}=\frac{e E}{m \omega^{\prime c}} \simeq 5.585\left(\frac{S \Delta f}{n}\right)^{1 / 2} \frac{D}{\delta f}
$$

where $S$ is the Hux density in $\mathrm{Jy}, f$ is the pulsar cmission frequency in $\mathrm{MHz}, \Delta f$ is the emission bandwidth in $\mathrm{MHz}, D$ is the pulsar distance in $\mathrm{kpc}, \delta$ is the size of emission region in $10^{*} \mathrm{~cm}$, and $n$ is the index of refraction. Using the measured microstructure properties of two pulsars, PSR 0950+08 (Cordes and Hankins 1979) and PSR 1133+16 (Bartel and Hankins 1982), Chian and Fennel (1983) estimated that the intensity of pulsar radio emissions in the source region should lie in the range $10^{-2} \leq \nu_{\mathrm{E}} \leq 10^{3}$. In the field of such strong waves, plasma particles in the pulsar magnetosphere may acquire weakly relativistic to moderately relativistic velocities. This is in contrast to strong low frequency pulsar magnetic dipole radiation for which $\nu_{E} \geq 10^{11}$ (Gunn and Ostriker 1971, Chian and Clemow 1975. Chian 1981. Chian 1982). It was demonstrated by Chian and Ken- nel (1983) that the nonlinearities arising from relativistic particle mass variation may excite a selfmodulational instability of strong electromagnetic waves in an electron-positron plasma. The resulting nonlinear wave envelopes can take the form of solitons or periodic wave trains. Chian and Kennel (1983) suggested that these nonlinear wave modulations may account for the formation of pulsar microstructures.

The purpose of this paper is to give a brief review of the nonlinear temporal model for formation of pulsar microstructures proposed by Chian and Kennel (1983) and discussed in a number of papers since. The next section reviews the theories of electromagnetic envelope nonlinear waves in electron-positron plasmas. A final section discusses the application of the nonlinear temporal model for observation of pulsar microstructures.

\section{Theories of envelope nonlinear waves}

Consider a modulated wave packet expressed by

$$
A(r, t)=\frac{1}{2}\left[a(r, t) e^{i(k \cdot r-\omega t)}+c . c .\right],
$$

where $a(r, t)$ represents the complex envelope of the modulated wave that varies slowly in time compared to the frequency of the fast varying carrier, i.e., $a^{-1} \partial a / \partial t \ll \omega$ and varies slowly in space compared to the wave vector of the carrier, i.e., $a^{-1} \partial a / \partial r \ll k$. The nonlinear evolution of a small but finite-amplitude envelope wave propagating in the $x$ direction can be described by the nonlinear Schrödinger equation-NLS (e.g., Hasegawa 1975)

$$
i \frac{\partial a}{\partial t}+P \frac{\partial^{2} a}{\partial x^{2}}+Q|a|^{2} a=0
$$


where the coefficient $P$ is related to the group dispersion

$$
P=\frac{1}{2} \frac{\partial v_{\mathrm{g}}}{\partial k}=\frac{1}{2} \frac{\partial^{2} \omega}{\partial k^{2}},
$$

and the coefficient $Q$ is related to (the negative of) the nonlinear frequency shift

$$
Q=-\frac{\partial \omega}{\partial|a|^{2}}
$$

Modulational instability of the nonlinear modulated wave occurs if

$$
P Q>0,
$$

namely, when the group dispersion and the nonlinear frequency shift have opposite signs. As the modulation grows, the instability can evolve to nonlinear stationary states such as envelope solitons, envelope holes and envelope wave trains that result from the balance between dispersion and nonlinearity.

In this paper, only weakly nonlinear theories will be discussed. Large amplitude theories of envelope strong waves in electron-positron plasmas have been formulated by Chian and Kennel (1983), Yu, Shukla and Rao (1984), Yu and Rao (1985) and Shukla et al. (1986).

\section{Cold unmagnetized plasma}

Chian and Kennel (1983) showed that in the limit $\nu_{\mathrm{E}} \ll 1$, strong electromagnetic waves, of the form given by eq.(2), in a cold unmagnetized electronpositron plasma can be described by the nonlinear Schrödinger equation [eq.(3)] with the coefficients eqs. (4) and (5) given by

$$
\begin{aligned}
P & =\frac{c^{2}}{2 \omega}, \\
Q & =\frac{e^{2} \Omega_{\mathrm{p}}^{2}}{4 \omega m^{2} c^{2}},
\end{aligned}
$$

for circular polarization, and

$$
\begin{aligned}
P & =\frac{c^{2}}{2 \omega}, \\
Q & =\frac{3 e^{2} \Omega_{\mathrm{p}}^{2}}{16 \omega m^{2} c^{2}},
\end{aligned}
$$

for linear polarization, where $\Omega_{\mathrm{p}}=\sqrt{2} \omega_{\mathrm{p}}, \omega_{\mathrm{p}}=$ $\left(4 \pi n_{0} e^{2} / m\right)^{1 / 2}$, and $m$ is the mass of electron and positron. The NLS derived by Chian and Kennel
(1983) only included the effect of relativistic mass variation induced by radiation intensity. Evidently, both eqs.(7) and (8) satisfy the condition of eq.(6) for excitation of modulational instability. In addition, Chian and Kennel (1983) determined the threshold and maximum growth rate for the instability and obtained the following nonlinear stationary solutions for NLS

$$
a=a_{0} \operatorname{sech}^{2}\left(\left|\frac{Q}{2 P}\right|^{1 / 2} a_{0} \xi\right)
$$

and

$$
a=a_{0} \mathrm{cn}^{2}\left(\left|\frac{Q}{2 P}\right|^{1 / 2} a_{0} \xi\right),
$$

where $\xi=x-V t$ and $V$ is the velocity of the envelope wave. Eqs.(9) and (10) describe envelope solitons and envelope nonlinear wave trains, respectively.

Mofiz, Angelis and Forlani $(1984,1985)$ extended the formulation of Chian and Kennel (1983) to the cases of constant and variable longitudinal particle velocity. In the case of constant longitudinal particle velocity, they showed that for circular polarization the coefficients $P$ and $Q$ of NLS are still given by equation (7) (Mofiz, de Angelis, and Forlani 1984); whereas for linear polarization they concluded that the nonlinear wave equation has periodic solutions but no localized solutions. In the case of variable longitudinal particle velocity, they showed that envelope solitons exist for both circular and linear polarizations, with the coefficients of NLS for both polarizations given by (Mofiz, de Angelis, and Forlani 1985)

$$
\begin{aligned}
P & =\frac{c^{2}}{2 \omega}, \\
Q & =\frac{\omega_{p}^{2}\left(\beta^{2}-1\right)}{4 \omega \gamma_{0}^{3}\left(\beta-\beta_{0}\right)^{2}},
\end{aligned}
$$

where $\beta=V / c, \beta_{0}=v_{x} / c$ as $\xi \rightarrow \pm \infty$, and $\gamma_{0}=\left(1-\beta_{0}^{2}\right)^{-1 / 2}$. The envelope soliton solution is described by

$$
a=a_{0} \operatorname{sech}\left(\left|\frac{Q}{2 P}\right|^{1 / 2} a_{0} \xi\right)\left\{\begin{array}{l}
\hat{y} \cos \theta \text { for LP } \\
(\hat{y} \cos \theta+\hat{z} \sin \theta) \text { for CP }
\end{array}\right.
$$

where the phase $\theta$ is given by

$$
\begin{aligned}
\theta(x, t)= & \frac{\omega \beta}{c} x-\left[\frac{1}{2}\left(1+\beta^{2}\right) \omega+\frac{\omega_{\mathrm{p}}^{2}}{2 \omega \gamma_{0}}\right. \\
& \left.-\frac{\omega_{\mathrm{p}}^{2}}{4 \omega} \frac{\beta^{2}-1}{2 \gamma_{0}^{3}\left(\beta-\beta_{0}\right)^{2}}\right] t .
\end{aligned}
$$




\section{Magnetized plasma}

Stenflo, Shukla and Yu (1985) considered the nonlinear propagation of circularly polarized electromagnetic waves parallel to the ambient magnetic field $B_{0} x$ in a cold electron-positron plasma. In addition to the relativistic particle mass modulation nonlinearity, they included the effects of the timederivative ponderomotive force, free-streaming particle motion, as well as second-order slow density perturbations. A set of nonlinear equations was derived to describe the modulational coupling of the radiation with electrostatic oscillations along the external magnetic field. Two cases were treated in the limit of strong magnetic field $\left(\omega_{c} \gg \omega\right.$, where $\left.\omega_{\mathrm{c}}=e B_{0} / m c\right)$. For the case $V^{2} \partial^{2} / \partial \xi^{2} \gg \omega_{\mathrm{p}}^{2}$, the set of coupled equations reduces to NLS with

$$
\begin{aligned}
P= & \frac{1}{2} \frac{\partial v_{\mathrm{g}}}{\partial k} \\
Q= & \frac{v_{\mathrm{g}} \omega_{\mathrm{p}}}{k c^{2} B_{0}^{2}}\left\{\frac{\omega^{2}\left[c^{-2}-V^{-2}(1-2 k V / \omega)^{2}\right]}{\omega_{\mathrm{c}}^{2}}\right. \\
& \left.-\frac{(1-k V / \omega)^{2}}{V^{2}}\right\}
\end{aligned}
$$

where for the Alfvén waves $v_{\mathrm{g}}$ is determined from the linear dispersion relation (Sakai and Kawata 1980b, Sakai and Kawata 1980a)

$$
\omega=k v_{\mathrm{A}}\left(1-\frac{k^{2} v_{\mathrm{A}}^{4} \omega_{\mathrm{p}}^{2}}{\omega_{\mathrm{c}}^{4} c^{2}}\right),
$$

with the Alfvén velocity given by

$$
v_{\mathrm{A}}=c\left(1+2 \omega_{\mathrm{p}}^{2} / \omega_{\mathrm{c}}^{2}\right)^{-1 / 2} .
$$

For the case $V^{2} \partial^{2} / \partial \xi^{2} \ll \omega_{\mathrm{p}}^{2}$, the set of coupled equations reduces to

$$
\begin{aligned}
i \frac{\partial E}{\partial t}+ & i\left(v_{\mathrm{g}}-I^{\prime}\right) \frac{\partial E}{\partial \xi}+\frac{1}{2} \frac{\partial v_{\mathrm{g}}}{\partial k} \frac{\partial^{2} E}{\partial \xi^{2}} \\
& +Q_{1}\left|E^{2}\right| E-Q_{2} E \frac{\partial^{2}}{\partial \xi^{2}}|E|^{2}=0
\end{aligned}
$$

where

$$
Q_{1}=\left[\frac{1}{c^{2}}-\frac{1}{V^{2}}\left(1-\frac{2 k V}{\omega}\right)^{2}\right] \frac{v_{\mathrm{g}} \omega^{2} \omega_{\mathrm{p}}^{2}}{k c^{2} B_{0}^{2} \omega_{\mathrm{c}}^{2}},
$$

and

$$
Q_{2}=\left(1-\frac{k V}{\omega}\right)^{2} \frac{v_{8}}{2 k c^{2} B_{0}^{2}} .
$$

Eq.(17) admits a cusped soliton solution (Litvak and Sergeev 1978). Mofiz and Podder (1987) and Mofiz et al. (1988) studied the propagation of nonlinear circularly polarized electromagnetic waves along the ambient magnetic field in an isothermal electron-positron plasma. Mofiz and Podder (1987) investigated the problem by assuming a quasistatic slow plasma response so that the radiation pressure is balanced by the ambipolar potential and thermal pressure. They showed that in a strongly magnetized plasma, the nonlinear envelope is governed by NLS with

$$
\begin{aligned}
P & =\frac{c^{2}}{2 \omega} \\
Q & =\frac{\omega_{\mathrm{p}}^{2}}{2 \omega \nu_{B}^{2}}\left(\frac{1}{\nu_{B}}+\frac{m c^{2}}{K T}\right),
\end{aligned}
$$

where $\nu_{B}=e B_{0} / m c \omega$. They found that the ambient magnetic field affects the envelope soliton with modification in the phase $\theta(x, t)$. For large $\nu_{B}$, they concluded that the height of the localized field is directly proportional to the strength of the ambient magnetic field $\nu_{B}$. Mofiz et al. (1988) investigated the problem by taking into consideration the slow plasma density perturbation due to the time-derivative ponderomotive force, relativistic mass modulation and the charge separation arising from higher-order nonlinearity. They showed that for $\omega_{c}^{2} \gg \omega^{2}$ the nonlinear evolution of the modulated wave is described by NLS with

$$
\begin{aligned}
& P=\frac{1}{2} \frac{\partial v_{\mathrm{g}}}{\partial k} \\
& Q=\frac{e^{2} \omega_{\mathrm{p}}^{2} \omega}{2 m^{2} c^{2} \omega_{\mathrm{c}}^{2}}\left[1+\frac{c^{2}}{v_{\mathrm{th}}^{2}-V^{2}}\left(1-\frac{2 k V}{\omega}\right)\right]
\end{aligned}
$$

where $v_{\text {th }}=(K T / m)^{1 / 2}$. They concluded that for $V^{2}<v_{\mathrm{th}}^{2}<\omega^{2} / k^{2}, Q$ is positive so that the condition of eq.(6) is met. Thus, NLS admits subthermal envelope solitons. The soliton width is seen to be independent of $B_{0}$, while the envelope amplitude is proportional to $B_{0}^{2}$.

Shukla (1985) derived a nonlinear Schrödinger equation for linearly polarized electromagnetic waves $(0$-mode $)$ propagating perpendicularly to the ambient magnetic field in a cold electron-positron plasma. They showed that in this case the coefficients of NLS are given by

$$
\begin{aligned}
P & =\frac{c^{2}}{2 \omega} \\
Q & =\frac{\omega_{\mathrm{p}}^{2}}{2 \omega}\left(\frac{c^{2} k^{2}}{\omega_{\mathrm{c}}^{2}}+\frac{3}{4}\right) .
\end{aligned}
$$




\section{Electron-positron-ion plasma}

Rizzato (1988) analyzed the oblique modulation of strong circularly polarized electromagnetic waves in a cold unmagnetized plasma made up of electrons, positrons and ions. The following nonlinear wave equation was derived for the normalized envelope amplitude $a$

$$
\frac{d^{2} a}{d \xi^{2}}=B a-C a^{3}
$$

where

$$
\begin{aligned}
B & =\frac{\delta^{\prime}}{\left(V^{2} / c^{2}-1\right) \beta_{x}^{2}-\beta_{z}^{2}} \\
C & =\frac{1-4 r(1-r)\left(c^{2} / \beta_{x}^{2} V^{2}\right)}{\left(V^{2} / c^{2}-1\right) \beta_{x}^{2}-\beta_{z}^{2}}
\end{aligned}
$$

with

$$
r=\frac{n_{0 \mathrm{p}}}{n_{0 \mathrm{p}}+n_{0 \mathrm{e}}}
$$

$\delta^{\prime}$ is the nonlinear frequency shift, $V=k c^{2} / \omega$ and $\xi=\beta_{x}(x-V t)+\beta_{z} z$. Eq.(23) admits a localized solution

$$
a=\left(\frac{2 B}{C}\right)^{1 / 2} \operatorname{sech} B^{1 / 2} \xi,
$$

if the following condition

$$
C \gg B>0
$$

is satisfied. Rizzato (1988) concluded that the possibility of localization of high frequency electromagnetic waves depends on the concentration of particles, the soliton velocity, and the angle between the direction of the envelope wave and the direction of the carrier wave. In particular, in the case of pure electron-positron plasma it was shown that no localized solution is possible, which is in contrast to the results derived by Mofiz, Angelis and Forlani (1985) from a similar formulation.

Rizzato, Schneider and Dillenburg (1988) studied the nonlinear modulation of circularly polarized electromagnetic waves propagating along the ambient magnetic field in a cold strongly magnetized electron-positron-ion plasma. In the limit $\omega_{\mathrm{c}} \gg \omega$, they derived the following nonlinear wave equation for the normalized envelope amplitude $a$

$$
\begin{aligned}
& \left(D \frac{V^{2}}{c^{2}}-1\right) \frac{d^{2} a}{d \xi^{2}}= \\
& \delta^{\prime} a+\left[1-\frac{4 c^{2}}{V^{2}}\left(1-\frac{2 k V}{\omega}\right)^{2} r(1-r)\right] \frac{a^{3}}{\nu_{B}^{4}}
\end{aligned}
$$

where $D=1+\omega_{0}^{2} / \omega_{\mathrm{c}}^{2}$ and $\omega_{0}^{2}=4 \pi e^{2}\left(n_{0 \mathrm{e}}+n_{0 \mathrm{p}}\right) / m$. Eq.(28) admits both compressive and rarefactive envelope soliton solutions provided the following condition for modulational instability is satisfied

$$
\frac{4 c^{2}}{V^{2}}\left(1-\frac{2 k V}{\omega_{ \pm}}\right)^{2} r(1-r)<1,
$$

where $\omega_{ \pm}$denotes the frequencies of the two different wave modes that appear in a magnetized electron-positron-ion plasma.

Note that in the nonlinear wave equations derived by Rizzato (1988) and Rizzato, Schneider and Dillenburg (1988), the NLS is modified by the presence of a $\partial^{2} a / \partial t^{2}$ term. They showed that, for high-velocity $(V \rightarrow c)$ solitons the temporal second derivative can be as important as the spatial second derivative.

\section{Relativistic plasma}

In the works reviewed so far, the plasma thermal energy was considered nonrelativistic ( $K^{\top} T \ll$ $m c^{2}$ ). Sakai and Kawata (1980a) derived the basic hydrodynamic equations valid for ultrarelativistic $\left(K T \gg m c^{2}\right)$ plasma. These equations were used by Mikhailovskii, Onishchenko and Tatarinov (1985a) and Mofiz (1989) to study the modulation of strong electromagnetic waves in a relativistic electron-positron plasma.

Mikhailovskii, Onishchenko and Tatarinov (1985a) considered the propagation of a nonlinear linearly-polarized Alfvén wave packet traveling along the ambient magnetic field in a strongly magnetized $\left(\omega^{2} \ll \omega_{c}^{2}\right)$ relativistic electron-positron plasma. They derived a NLS for the nonlinear Alfvén wave packet, with the coefficients given by

$$
P=-\frac{3 k \omega_{\mathrm{p}}^{2} V^{3}}{\omega_{\mathrm{c}}^{4}\left(1+2 \omega_{\mathrm{p}}^{2} / \omega_{\mathrm{c}}^{2}\right)}
$$

and

$$
Q=-\frac{3 k \omega^{2} V}{2 \omega_{\mathrm{c}}^{2}\left(1+2 \omega_{\mathrm{p}}^{2} \omega_{\mathrm{c}}^{2}\right)} \frac{1-c_{\mathrm{s}}^{2} / c^{2}}{1-c_{\mathrm{s}}^{2} / V^{2}}\left(1-\frac{V^{2}}{c^{2}}\right),
$$

where $c_{s}=\left(K T n_{0} / \rho_{0}\right)^{1 / 2}$ is the acoustic velocity, and $\omega_{\mathrm{p}}$ and $\omega_{\mathrm{c}}$ are the relativistic plasma and cyclotron frequencies, respectively.

Mofiz (1989) studied the nonlinear propagation of circularly polarized electromagnetic waves along the ambient magnetic field in a relativistic electronpositron plasma. He showed that the nonlinear modulation is governed by NLS with the coefficients given by 


$$
\begin{aligned}
P= & \frac{c^{2}}{2 \nu} . \\
Q= & \frac{1}{8 \pi} \frac{\dot{2}_{p}^{2}}{\omega^{2}-\nu_{c}^{2}}\left[\frac{1}{K^{2} T}-\frac{1}{2\left(P_{0}+E\right)}\right] \\
& \times\left[1+\frac{2 k V \omega_{c}^{2}}{\omega\left(\omega^{2}-\omega_{c}^{2}\right)}\right]
\end{aligned}
$$

where $P_{0}$ and $E$ denote the background plasma pressure and mass energy density, respectively. In the limit $K^{\prime} T>P_{0}+E$, the NLS has the solution

$$
|E|^{2}=E_{0}^{2} \csc ^{2}(\mu \xi)
$$

with

$$
\mu=\left[k^{\prime}\left(v_{\mathrm{g}}+P k^{\prime}\right)-\omega^{\prime}\right] / P^{1 / 2}
$$

where $k^{\prime}$ and $\omega^{\prime}$ are the wave number and frequency of the envelope wave, respectively. Eq.(33) describes a soliton which is cusped at the center, where $\partial E / \partial \xi$ becomes infinite. Thus the electric or magnetic fields appear as isolated spikes.

Kinetic theories of modulation of strong electromagnetic waves in a relativistic electron-positron plasma have been developed by Pataraya and Melikidze (1980), Lakhina and Buti (1981), Mikhailovskii, Orishchenko and Tatarinov (1985b) and Mikhailovskii, Onishchenko and Smolyakov (1985).

Lakhina and Buti (1981) derived a NLS for linearly polarized electromagnetic waves propagating in an unmagnetized ultrarelativistic electronpositron plasma for an isotropic distribution function, with the coefficients given by

$$
\begin{aligned}
P & =\frac{3 c^{2}}{\omega}, \\
Q & =\frac{\omega_{\mathrm{p}}^{2} m e^{2} c^{4}}{210 K^{-3} T^{3} \omega^{3}} .
\end{aligned}
$$

Mikhailovskii, Onishchenko and Tatarinov (1985b) and Mikhailovskii, Onishchenko and Smolyakov (1985) derived a NLS for linearly polarized Alfvén wave propagating along the ambient magnetic field in a relativistic electron-positron plasma, which for the case of a one-dimensional distribution function and a moving plasma has the following coefficients

$$
\begin{aligned}
P & =-\frac{k c^{3} \omega_{\mathrm{p}}^{2}}{8 \gamma_{\mathrm{p}} \omega_{\mathrm{c}}^{4}}, \\
Q & =-e \frac{3 k c \omega_{\mathrm{p}}^{4}}{16 \gamma_{\mathrm{p}}^{2} \omega_{\mathrm{c}}^{4}},
\end{aligned}
$$

where the distribution function is assumed to be a step function: $f\left(p_{\|}\right)=n_{0} / p_{0}$ for $0 \leq p_{\|} \leq p_{0}$, where $p_{0}=\gamma_{\mathrm{p}} m c$ and $\gamma_{\mathrm{p}} \gg 1 ; \omega_{\mathrm{p}}$ and $\omega_{\mathrm{c}}$ are the nonrelativistic plasma and cyclotron frequencies, respectively.

The effects of wave-particle interaction, such as nonlinear Landau damping and cyclotron resonance, on the modulation of strong electromagnetic waves in relativistic electron-positron plasmas have been treated by Pataraya and Melikidze (1980) and Mikhailovskii, Onishchenko and Tatarinov (1985b). Pataraya and Melikidze (1980) derived a modified NLS for both linearly and circularly polarized electromagnetic waves in an unmagnetized relativistic electron-positron plasma by taking into account the nonlinear Landau damping, and showed that envelope soliton solutions exist. Mikhailovskii, Onishchenko and Tatarinov (1985b) discussed the modification of the NLS due to cyclotron-resonant particles and showed that envelope Alfvén solitons can be excited in a relativistic electron-positron plasma by a beam of high-energy particles.

\section{Discussion}

As shown in the previous section, the modulational instability of strong electromagnetic waves can be excited in electron-positron plasmas under a variety of physical conditions expected in pulsar magnetospheres, which confirms the nonlinear temporal model originally proposed by Chian and Kennel (1983). According to this model, as represented by eq.(2), the high frequency coherent pulsar radio emission with a fast-time scale $2 \pi / \omega$ can be modulated by a slowly time-varying envelope $a(x, t)$ due to nonlinear effects. The resulting nonlinear envelopes may account for formation of pulsar microstructures.

The theories in the previous section indicate that the end products of the modulational instability may be nonlinear stationary solutions of either envelope solitons or envelope nonlinear wave trains. This is consistent with the observed features of pulsar microstructures. Hankins and Boriakoff (1978) showed that the observed pulsar microstructures can be put into two categories, intermittent type or quasi-periodic type, according to their intensity structures. Most micropulses are of the intermittent type, which have bursts of strong emission interspersed with sections of longitude where the signal returns abruptly to the system noise level. Occasionally, quasi-periodic strings of micropulses are detected. The intermittent micropulses can be interpreted by the nonlinear temporal model as a collection of envelope solitons with randomly fluc- 
tuating amplitudes, whereas the quasi-periodic micropulses can be explained as periodic envelope nonlinear wave trains or a sequence of envelope solitons with very little variation in their peak amplitudes. Note that the temporal profiles of pulsar microstructures are usually quite spiky. Physically, the steep structures may be attributed to the tendency of wave steepening and wave breaking in the presence of nonlinearities (Chian 1979, Chian 1989, Yu, Shukla, and Rao 1984).

The nonlinear envelope solutions discussed above can be used to give the estimates of microstructure characteristics. According to the nonlinear temporal model, the envelope spatial pulse width is

$$
d=\left|\frac{2 P}{Q}\right|^{1 / 2} \frac{1}{a_{0}},
$$

the number of wave crests in a given modulation is

$$
N=d / \lambda=\left|\frac{2 P}{Q}\right|^{1 / 2} \frac{k}{2 \pi a_{0}},
$$

and the temporal pulse width is

$$
\tau=d / V=\left|\frac{2 P}{Q}\right|^{1 / 2} \frac{1}{a_{0} V} .
$$

From the observed temporal pulse widths of pulsar microstructures ( $\sim \mu \mathrm{s}$ to $1 \mathrm{~ms})$ and the frequency range of pulsar radio emission $(\sim 100 \mathrm{MHz}$ to $1 \mathrm{GHz}$ ) we see that $N$ varies from $\sim 10^{2}$ to $10^{6}$. Theoretical estimates based on eqs.(37)-(39) give results which are consistent with the observations (Chian and Kennel 1983, Mofiz, de Angelis, and Foriani 1985, Shukla 1985, Mofiz and Podder 1987, Mikhailovskii, Onishchenko, and Tatarinov 1985a, Mikhailovskii, Onishchenko, and Smolyakov 1985). One important feature of the nonlinear temporal model, as evidenced in eq.(37), suggests that the higher the micropulse intensity, the narrower the pulse width. Such behavior was shown by Bartel, Sieber and Graham (1980) to hold true for pulsar subpulses and was suggested by Ferguson (1981) to be true also for micropulses. It should be interesting for pulsar observers to verify this property across the entire range of microstructure time scales.

The Alfvén waves, which can be generated by a high energy beam of particles or decay of Langmuir waves, are the most likely candidate to go out of the pulsar magnetosphere and to be interpreted as the high-frequency coherent radio emission observed on the Earth (Mikhailovskii, Onishchenko and Tatarinov 1985a, b; Mikhailovskii, Onishchenko and Smolyakov 1985). Therefore, the nonlinear temporal model suggests that pulsar microstructures are Alfvén envelope solitons or Alfvén envelope nonlinear wave trains generated through the modulational interaction of high frequency coherent Alfvén wave packets with the pulsar magnetosphere.

Acknowledgments: I wish to thank I. S. Batista and J. Braga for valuable assistance in the preparation of this manuscript. 